\title{
Study of Tachyonic Field and its statefinder diagnostics in Various Scenarios of The Anisotropic Universe
}

\author{
Chayan Ranjit ${ }^{1, *}$ and Surajit Chattopadhyay ${ }^{2,+}$ \\ ${ }^{1}$ Department of Mathematics, Seacom Engineering College, Howrah - 711 302, India. \\ ${ }^{2}$ Department of Computer Application (Mathematics Section), \\ Pailan College of Management and Technology, \\ Bengal Pailan Park, Kolkata-700 104, India.
}

(Dated: October 18, 2018)

\begin{abstract}
In the present work, we have considered N-dimensional Einstein field equations in which 4-dimensional space-time is described by a FRW metric and that of the extra d-dimensions by an Euclidean metric. Considering the universe filled with tachyonic field we have reconstructed the potential $V(\phi)$ corresponding to the field reconstructions in an anisotropic universe under Emergent-Power law, Emergent-Intermediate, Emergent-Logamediate and Logamediate-Intermediate scenarios. The statefinder parameters have been investigated in all of the said scenarios.
\end{abstract}

\section{INTRODUCTION}

The two independent observational signals on distant Type Ia supernovae (SNIa) in 1998 have revealed the speeding up of our universe. This acceleration implies that if the theory of Einstein's gravity is reliable on cosmological scales, then our universe is dominated by a mysterious form of matter. This unknown component possesses some remarkable features, for instance it is not clustered on large length scales and its pressure must be negative in order to be able to drive the current acceleration of the universe [1]. This matter content is dubbed as "dark energy" (DE) that occupies about $70 \%$ of today's universe. Reviews on DE include [1], 2], [3] and [4]. A chronological history of the research on DE is discussed in [4]. The basic characteristic of DE is that its equation of state (EOS) parameter $w=p / \rho$, where $\rho$ is the energy density and $p$ is the pressure that has a negative value. The simplest candidate of dark energy is a tiny positive cosmological constant [2] corresponds to a fluid with a constant equation of state $w=-1$. However, as is well known, it is plagued by the so-called "cosmological constant problem" and "coincidence problem" [2]. Other

*Electronic address: chayanranjit@gmail.com

${ }^{\dagger}$ Electronic address: surajit‘2008@yahoo.co.in, surajit.chattopadhyay@rediffmail.com 
dark energy models include quintessence [5], phantom [6], quintom [1], Chaplygin gas [7], tachyon [8], hessence [9] etc.

The weakness of the gravitational force has been successfully explained by postulating the existence of extra dimensions [10]. Today, there is a large variety of promising models and theories which suggest the existence of more than three spatial dimensions. Most notably, string theory suggests the existence of seven additional spatial dimensions [11]. As our space-time is explicitly four dimensional in nature so the 'hidden' dimensions must be related to the dark matter and dark energy which are also 'invisible' in nature. Model of higher dimension was proposed by Kaluza and Klein [12, 13] who tried to unify gravity with electromagnetic interaction by introducing an extra dimension which is basically an extension of Einstein general relativity in 5D. The activities of extra dimensions also verified from the STM theory [14] proposed recently by Wesson et al. [15]. The importance of extra dimension in cosmology has been discussed by many authors. In some analysis the metric is assumed to be uniform in extra dimension [18, 19]. For the metric having non-trivial dependence the extra dimension, the analysis on inflation may be altered [20, 21]. Panigrahi and Chatterjee [22] have found that the inflationary scenario is possible for inhomogeneous extra dimensional model and for the homogeneous case an initially decelerating universe starts accelerating undergoing a flip. Ibánez and Verdaguer [23] have obtained a set of solutions of Einstein's equation in an N-dimensional vacuum model and also homogeneous solutions with expanding 3-dimensional isotropic space. The 4D perfect fluid solutions in flat universe model have been obtained by Krori et al. [24], Gleiser and Diaz [25], using a higher dimensional anisotropic cosmology (Bianchi-I) which are compatible with contraction of all the extra dimensions. In 26] Paul has considered the theories of imperfect fluid such as Eckart, EIT, TIS and FIS to obtain the cosmological solutions for flat FRW with extra dimensions by Kasner type Euclidean metric and present an analysis of a n-dimensional vacuum Einstein's field equations. In [27] Gorbunov and Sibiryanov have proposed a cosmological model of self accelerated brane universe with warped extra dimension. In Peng et al. [28] extended the direct quantum approach to the FRW cosmology from 4D to 5D and obtained a Hamiltonian formulation for a wave like 5D FRW cosmology. In Panigrahi et al. [29] have shown a scenario in-homogeneous 5D space time which behave a decelerating expansion in the early epoch along with an accelerating situation at the present line without introducing any external quintessence-like scalar field in the presence of extra dimension.

In the present work, we have considered N-dimensional Einstein field equations in which 4dimensional space-time is described by a FRW metric and that of the extra d-dimensions by an Euclidean metric. Considering the universe filled with tachyonic field we have reconstructed 
the potential $V(\phi)$ corresponding to the field reconstructions in an anisotropic universe. A rolling tachyon has an interesting equation of state whose parameter smoothly interpolates between -1 and 0 [30]. This has led to several attempts to construct viable cosmological models using the tachyon as a suitable candidate for the inflaton at high energy [2], [31], [32], [39], [16], [17]. In a flat FRW background the energy density and pressure of tachyon are given by [2]

$$
\rho=\frac{V}{\sqrt{1-\dot{\phi}^{2}}} ; \quad p=-V \sqrt{1-\dot{\phi}^{2}}
$$

where $V$ and $\phi$ denote the potential and tachyonic field respectively. The tachyonic matter might provide an explanation for inflation at the early epochs and could contribute to some new form of cosmological dark matter at late times [32]. In isotropic FRW universe, the tachyonic field was studied by [33], where it was observed by studying the statefinder diagnostics [34] that the tachyonic interpolates between dust and $\Lambda$ CDM stages of the universe. In the present paper we are going to consider the scale factors $a(t)$ and $b(t)$ in the following forms:

1. $a(t)=A\left(\beta+e^{\alpha t}\right)^{m}$ with $A>0, \quad \alpha>0, \quad \beta>0, \quad m>1$ (emergent expansion) [35, 43].

2. $a(t)=\exp \left(A(\ln t)^{m}\right)$ with $A m>0, \quad m>1$ (logamediate expansion) [36, 42].

3. $b(t)=\exp \left(B t^{n}\right)$ with $B>0 ; 0<n<1$ (intermediate expansion) [36, 42].

4. $b(t)=\exp \left(B(\ln t)^{n}\right)$ with $B n>0, \quad n>1$ (logamediate expansion) [36, 42].

5. $b(t) \propto t^{n}$ with $n>1$ (power law expansion) [2].

Some authors first choose the scale factor in power law, exponential or in other forms and then find out other variables with some conditions under these solutions. This 'reverse way of investigations had earlier been used extensively by [37] who chose various forms of scale factor and then found out the other variables from the field equations. Subsequently, this approach has been adopted by [38], who clearly stated "This is not the ideal way to find out the dynamics of the universe, as here the dynamics is assumed and then the fields are found out without any reference to the origin of the field. But in the absence of more rigorous ways, this kind of investigations collectively might finally indicate towards the path where one really has to search". In another study, reference [31] assumed scale factor in the power law form to model the potential by an inverse square law in terms of the tachyon field.

In the present work we have adopted this "reverse approach" to reconstruct the potential of the 
tachyonic field in the anisotropic universe. Subsequently, we have investigated how the statefinder parameters $\{r, s\}$ behave in this case. We have chosen combinations of choices of scale factors and investigated different scenarios under the assumption that the universe is filled with tachyonic field. The basic equations are discussed in the following section.

\section{BASIC EQUATIONS}

In the present work we consider homogeneous and anisotropic $N$-dimensional space-time model described by the line element[41]

$$
d s^{2}=d s_{F R W}^{2}+\sum_{i=1}^{d} b^{2}(t) d x_{i}^{2}
$$

where $d$ is the number of extra dimensions $(d=N-4)$ and $d s_{F R W}^{2}$ represents the line element of the FRW metric in four dimensions is given by

$$
d s_{F R W}^{2}=-d t^{2}+a^{2}(t)\left[\frac{d r^{2}}{1-k r^{2}}+r^{2}\left(d \theta^{2}+\sin ^{2} \theta d \phi^{2}\right)\right]
$$

where $a(t)$ and $b(t)$ are the functions of $t$ alone represent the scale factors of 4 -dimensional space time and extra $d$-dimensions respectively. Here $k(=0, \pm 1)$ is the curvature index of the corresponding 3-space, so that the above Universe is described as flat, closed and open respectively.

The Einstein's field equations for the above non-vacuum higher dimensional space-time symmetry are

$$
\begin{gathered}
3\left(\frac{\dot{a}^{2}+k}{a^{2}}\right)=\frac{\ddot{D}}{D}-\frac{d^{2}}{8} \frac{\dot{b}^{2}}{b^{2}}+\frac{d}{8} \frac{\dot{b}^{2}}{b^{2}}+\rho \\
2 \frac{\ddot{a}}{a}+\frac{\dot{a}^{2}+k}{a^{2}}=\frac{\dot{a}}{a} \frac{\dot{D}}{D}+\frac{d^{2}}{8} \frac{\dot{b}^{2}}{b^{2}}-\frac{d}{8} \frac{\dot{b}^{2}}{b^{2}}-p
\end{gathered}
$$

and

$$
\frac{\ddot{b}}{b}+3 \frac{\dot{a}}{a} \frac{\dot{b}}{b}+\frac{\dot{D}}{D} \frac{\dot{b}}{b}-\frac{\dot{b}^{2}}{b^{2}}=-\frac{p}{2}
$$




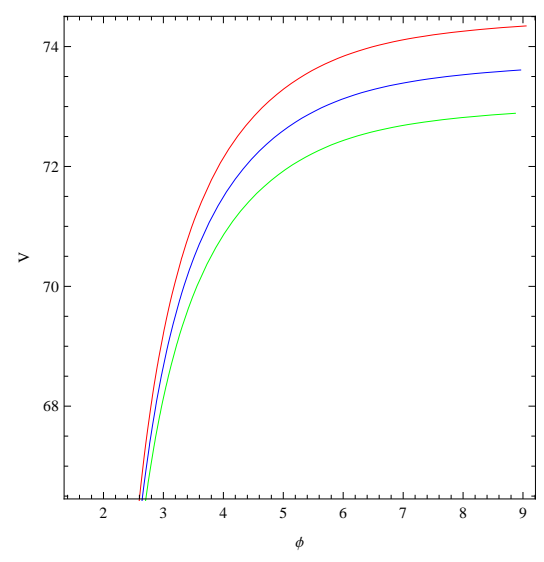

Fig.1

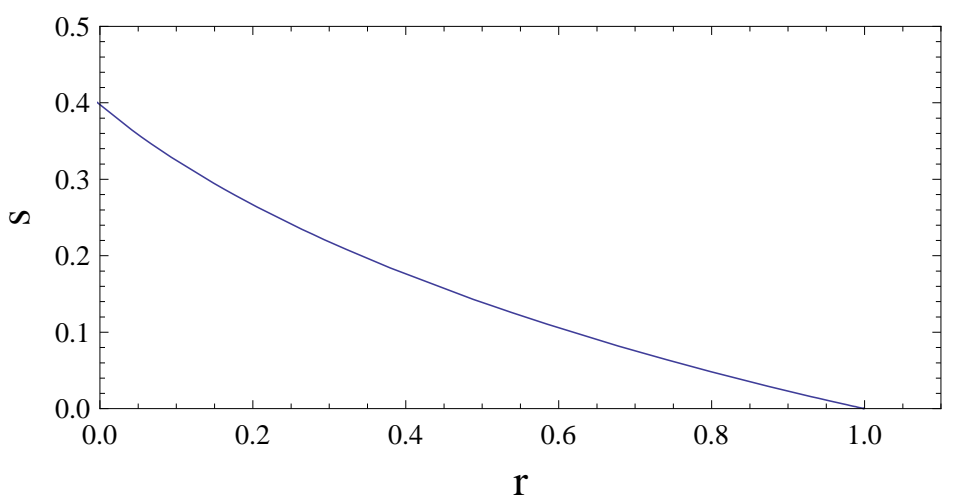

Fig.2

Fig. 1 shows the variations of $V$ against $\phi$, for $A=1, B=2, k=-1,0,1, \alpha=2.5, \beta=3, m=2, n=3, d=5$ and Fig. 2 shows the variation of the statefinder parameters $r$ against $s$ for $A=2, B=3, \alpha=3, \beta=2, m=3, n=2, d=5$ in the case of

Tachyonic field for Emergent-Powerlaw Scenario.

where $\rho$ and $p$ are energy density and isotropic pressure respectively. Here we choose here $8 \pi G=c=1$ and $D^{2}=b^{d}(t)$, so we have $\frac{\dot{D}}{D}=\frac{d}{2} \frac{\dot{b}}{b}$ and $\frac{\ddot{D}}{D}=\frac{d}{2} \frac{\ddot{b}}{b}+\frac{d^{2}-2 d}{4} \frac{\dot{b}^{2}}{b^{2}}$.

Sahni et al [34] proposed the trajectories in the $\{\mathrm{r}, \mathrm{s}\}$ plane corresponding to different cosmological models to depict qualitatively different behavior. Since this parameters are dimensionless so they allow us to characterize the properties of dark energy in a model independently. The statefinder diagnostic along with future SNAP observations may perhaps be used to discriminate between different dark energy models. The above statefinder parameters are given by [34]:

$$
r=1+3 \frac{\dot{H}}{H^{2}}+\frac{\ddot{H}}{H^{3}} \quad \text { and } \quad s=\frac{r-1}{3\left(q-\frac{1}{2}\right)}
$$

where $q$ is the deceleration parameter defined by $q=-1-\frac{\dot{H}}{H^{2}}$. In the anisotropic universe under consideration, the Hubble parameter $H$ is given by $H=\frac{1}{d+3}\left(3 \frac{\dot{a}}{a}+d \frac{\dot{b}}{b}\right)$. In this paper we have reconstructed the statefinder parameters for different scenarios to be discussed in the subsequent sections. Finally we graphically analyzed $\{r, s\}$ trajectory for various scenarios.

\section{- Emergent-Power law Scenario:}




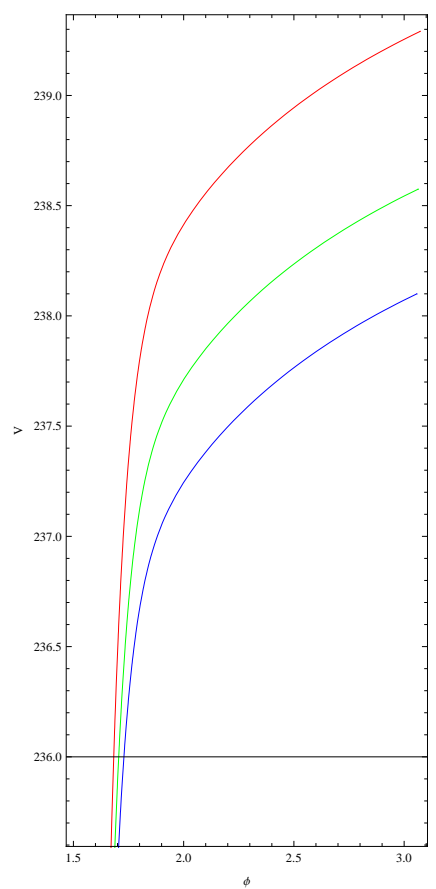

Fig.3

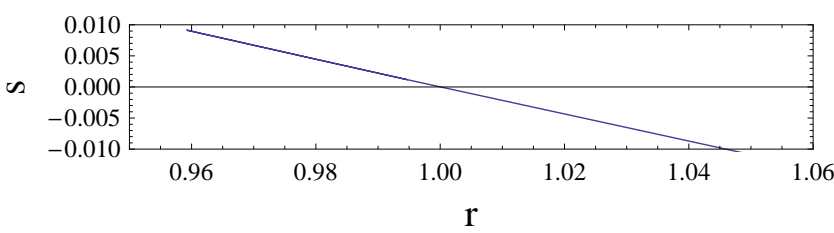

Fig.4

Fig. 3 shows the variations of $V$ against $\phi$, for $A=2, B=1, k=-1,0,1, \alpha=3, \beta=2, m=3, n=.8, d=3$ and Fig. 4 shows the variation of the statefinder parameters $r$ against $s$ for $A=2, B=3, \alpha=2, \beta=7, m=2, n=0.5, d=5$ in the case of Tachyonic field for Emergent-Intermediate Scenario.

In this section we consider a combination of scale factors $a$ and $b$ in the emergent and power laws form respectively. As we are considering anisotropic universe, this combination appears feasible. We name this scenario as "emergent-power law scenario". In this scenario we consider $a(t)$ and $b(t)$ as follows:

$$
a(t)=A\left(\beta+e^{\alpha t}\right)^{m} \text { and } b(t)=B t^{n}
$$

Using equations (4)-(6), we can find the expressions for $V(\phi)$ and $\phi$ as

$$
\begin{aligned}
& V(t)=\frac{1}{2 \sqrt{2}} \sqrt{\left(-\frac{d n(d n+n-4)}{8 t^{2}}+\frac{3 e^{2 \alpha t} m^{2} \alpha^{2}}{\left(e^{\alpha t}+\beta\right)^{2}}+\frac{3 k\left(\beta+e^{\alpha t}\right)^{-2 m}}{A^{2}}\right)} \\
& \times \sqrt{\left(\frac{8 k\left(e^{\alpha t}+\beta\right)^{-2 m}}{A^{2}}+\frac{8 e^{\alpha t} m \alpha^{2}\left(3 e^{\alpha t} m+2 \beta\right)}{\left(e^{\alpha t}+\beta\right)^{2}}+\frac{d n\left(n-d n-\frac{4 e^{\alpha t} m t \alpha}{e^{\alpha t}+\beta}\right)}{t^{2}}\right)}
\end{aligned}
$$




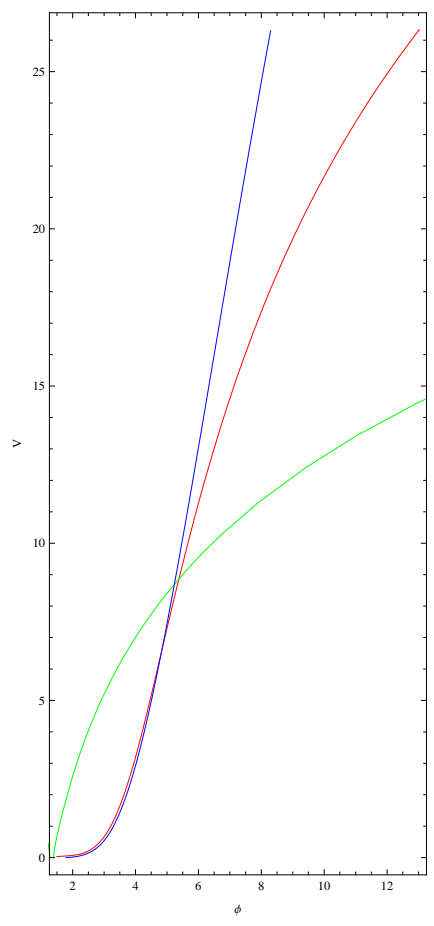

Fig.5

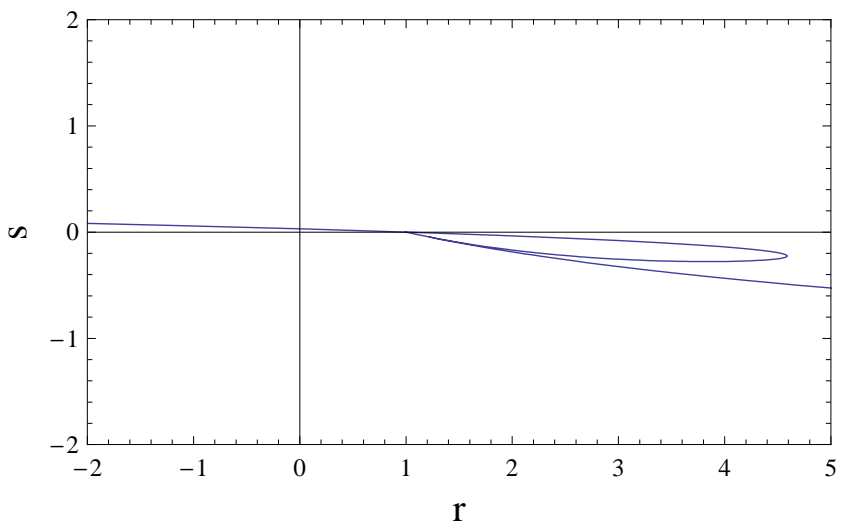

Fig.6

Fig. 5 shows the variations of $V$ against $\phi$, for

$A=2, B=1, k=-1,0,1, \alpha=0.1, \beta=1, m=0.3, n=5, d=3$ and Fig. 6 shows the variation of the statefinder parameters $r$ against $s$ for $A=2, B=3, \alpha=3, \beta=2, m=3.5, n=2, d=5$ in the case of

Tachyonic field for Emergent-Logamediate Scenario.

$$
\phi(t)=\int \sqrt{1+\frac{\frac{d^{2} n^{2}}{t^{2}}-\frac{8 k\left(e^{\alpha t}+\beta\right)^{-2 m}}{A^{2}}-\frac{8 e^{\alpha t} m \alpha^{2}\left(3 e^{\alpha t} m+2 \beta\right)}{\left(e^{\alpha t}+\beta\right)^{2}}+\frac{d n\left(n-d n-\frac{4 e^{\alpha t} m t \alpha}{e^{\alpha t}+\beta}\right)}{t^{2}}}{8\left(-\frac{d n(d n+n-4)}{8 t^{2}}+\frac{3 e^{2 \alpha t} m^{2} \alpha^{2}}{\left(e^{\alpha t}+\beta\right)^{2}}+\frac{3 k\left(\beta+e^{\alpha t}\right)^{-2 m}}{A^{2}}\right)}} d t
$$

Using equation (7), we can find the expressions for $r$ and $s$ as

$$
\begin{gathered}
r=1-\frac{3(d+3)\left(-3 e^{\alpha t} m t^{2} \alpha^{2} \beta+d n\left(e^{\alpha t}+\beta\right)^{2}\right)}{\left(e^{\alpha t}(d n+3 m t \alpha)+d n \beta\right)^{2}}+\frac{(3+d)^{2}\left(3 e^{\alpha t} m t^{3} \alpha^{3} \beta\left(-e^{\alpha t}+\beta\right)+2 d n\left(e^{\alpha t}+\beta\right)^{3}\right)}{\left(e^{\alpha t}(d n+3 m t \alpha)+d n \beta\right)^{3}} \\
s=\left[\frac{\left(-3(3+d)\left(e^{\alpha t}(d n+3 m t \alpha)+d n \beta\right)\left(-3 e^{\alpha t} m t^{2} \alpha^{2} \beta+d n\left(e^{\alpha t}+\beta\right)^{2}\right)+(3+d)^{2}\right.}{\left(3\left(e^{\alpha t}(d n+3 m t \alpha)+d n \beta\right)^{3}\right.}\right. \\
\left.\frac{\left.\times\left(3 e^{\alpha t} m t^{3} \alpha^{3} \beta\left(-e^{\alpha t}+\beta\right)+2 d n\left(e^{\alpha t}+\beta\right)^{3}\right)\right)}{\left.\times\left(-\frac{3}{2}+\frac{(3+d)\left(-3 e^{\alpha t} m t^{2} \alpha^{2} \beta+d n\left(e^{\alpha t}+\beta\right)^{2}\right)}{\left(e^{\alpha t}(d n+3 m t \alpha)+d n \beta\right)^{2}}\right)\right)}\right]
\end{gathered}
$$




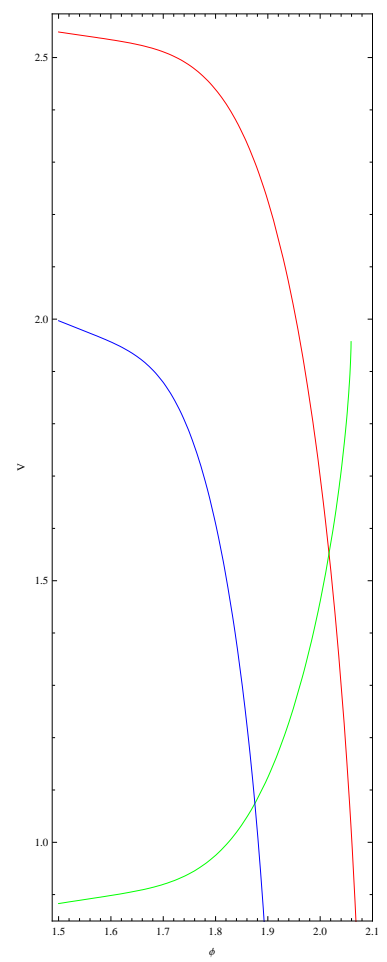

Fig.7

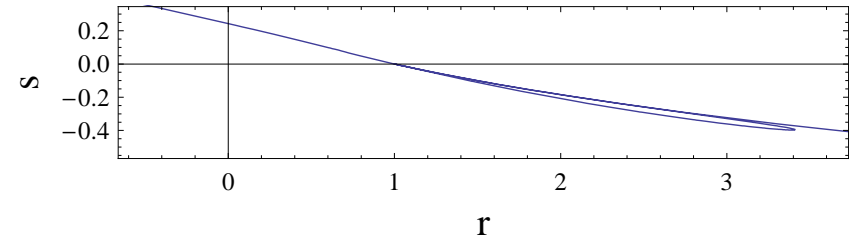

Fig. 8

Fig. 7 shows the variations of $V$ against $\phi$, for $A=2, B=1, k=-1,0,1, m=10, n=0.9, d=3$ and Fig. 8 shows the variation of the statefinder parameters $r$ against $s$ for $A=2, B=3, m=8, n=0.8, d=5$ in the case of Tachyonic field for Logamediate-Intermediate Scenario.

The above forms of statefinder parameters pertain to the emergent-power law scenario. The statefinder parameters derived above are plotted as $r-s$ trajectory in the figure 2 and the potential $V$ corresponding to this scenario is plotted against the scalar field $\phi$ in figure 1 .

\section{- Emergent-Intermediate Scenario:}

In this section we consider a combination of scale factors $a$ and $b$ in the forms of emergent and intermediate respectively. We dub this scenario as "Emergent-Intermediate Scenario". Thus, to describe this scenario we consider $a(t)$ and $b(t)$ as follows:

$$
a(t)=A\left(\beta+e^{\alpha t}\right)^{m} \text { and } b(t)=\exp \left(B t^{n}\right)
$$

Using equations (4)-(6), we can find the expressions for $V(\phi)$ and $\phi$ as 


$$
\begin{aligned}
& V(t)=\frac{1}{8} \sqrt{-\frac{e^{-2 A(\ln t)^{m}}}{t^{4}(\ln t)^{2}}\left(\left(24 k t^{2}-B d e^{2 A(\ln t)^{m}} n t^{n}\left(-4+n\left(4+B(d+1) t^{n}\right)\right)\right)(\ln t)^{2}+24 A^{2} e^{2 A(\ln t)^{m}} m^{2}(\ln t)^{2 m}\right)} \\
& \times \sqrt{\left(-8 e^{-2 A(\ln t)^{m}} k t^{2}+B^{2}(-1+d) d n^{2} t^{2 n}+4 A m(\ln t)^{m-2}\left(4-4 m+\left(4+B d n t^{n}\right)(\ln t)-6 A m(\ln t)^{m}\right)\right)}
\end{aligned}
$$

$$
\begin{gathered}
\phi(t)=\int \sqrt{1+\frac{e^{2 A(\ln t)^{m}}(\ln t)^{2}\left(-8 e^{-2 A(\ln t)^{m}} k t^{2}+B^{2}(-1+d) d n^{2} t^{2 n}+4 A m(\ln t)^{m-2}\right.}{\left(24 k t^{2}-B d e^{2 A(\ln t)^{m}} n t^{n}\left(-4+n\left(4+B(d+1) t^{n}\right)\right)(\ln t)^{2}+24 A^{2} e^{2 A(\ln t)^{m}} m^{2}(\ln t)^{2 m}\right.}} \\
\frac{\frac{\left.\times\left(4-4 m+\left(4+B d n t^{n}\right)(\ln t)-6 A m(\ln t)^{m}\right)\right)}{15)}}{d t}
\end{gathered}
$$

Using equation (7), we can find the expressions for $r$ and $s$ as

$$
\begin{gathered}
r=1+\frac{3(d+3)\left(B d(-1+n) n t^{n}(\ln t)^{2}+3 A m(-1+m-\ln t)(\ln t)^{m}\right)}{\left(B d n t^{n} \ln t+3 A m(\ln t)^{m}\right)^{2}} \\
+\frac{(3+d)^{2}\left(B d n(2+(-3+n) n) t^{n}(\ln t)^{3}+3 A m(\ln t)^{m}(2+(-3+m) m+\ln t(3-3 m+2 \ln t))\right)}{\left(B d n t^{n} \ln t+3 A m(\ln t)^{m}\right)^{3}} \\
s=\left[\frac{-\left(( 3 + d ) \left(3\left(B d n t^{n} \ln t+3 A m(\ln t)^{m}\right)\left(B d(-1+n) n t^{n}(\ln t)^{2}+3 A m(-1+m-\ln t)(\ln t)^{m}\right)\right.\right.}{3\left(B d n t^{n} \ln t+3 A m(\ln t)^{m}\right)^{3}\left(\frac{3}{2}+\frac{(d+3)\left(B d(-1+n) n t^{n}(\ln t)^{2}+3 A m(-1+m-\ln t)(\ln t)^{m}\right)}{\left(B d n t^{n} \ln t+3 A m(\ln )^{m}\right)^{2}}\right)}\right. \\
\frac{\left.\left.+(3+d)\left(B d n(2+(-3+n) n) t^{n}(\ln t)^{3}+3 A m(\ln t)^{m}(2+(-3+m) m+\ln t(3-3 m+2 \ln t))\right)\right)\right)}{}
\end{gathered}
$$

The forms of potential and $r-s$ trajectory derived in this scenario are presented in figures 3 and 4 respectively.

\section{- Emergent-Logamediate Scenario:}

In this section we consider the scale factor $a$ in emergent form and $b$ in the logamediate form. We dub this scenario as "Emergent-Logamediate Scenario". Thus, in this scenario we consider $a(t)$ and $b(t)$ as follows:

$$
a(t)=A\left(\beta+e^{\alpha t}\right)^{m} \text { and } b(t)=\exp \left(B(\ln t)^{n}\right)
$$


Using equations (4)-(6), we can find the expressions for $V(\phi)$ and $\phi$ as

$$
\begin{aligned}
& V(t)=\frac{1}{8} \sqrt{\left(24\left(\frac{e^{2 \alpha t} m^{2} \alpha^{2}}{\left(e^{\alpha t}+\beta\right)^{2}}+\frac{k\left(e^{\alpha t}+\beta\right)^{-2 m}}{A^{2}}\right)-\frac{B d n(\ln t)^{n-2}(4(n-1)-4 \ln t)+B(1+d) n(\ln t)^{n}}{t^{2}}\right)} \\
& \times \sqrt{\left(\frac{8 k\left(e^{\alpha t}+\beta\right)^{-2 m}}{A^{2}}+\frac{8 e^{\alpha t} m \alpha^{2}\left(3 e^{\alpha t} m+2 \beta\right)}{\left(e^{\alpha t}+\beta\right)^{2}}-\frac{B d n(\ln t)^{n-2}\left(4 e^{\alpha t} m t \alpha \ln t+B(-1+d) n\left(e^{\alpha t}+\beta\right)(\ln t)^{n}\right)}{t^{2}\left(e^{\alpha t}+\beta\right)}\right)}
\end{aligned}
$$

$$
\phi(t)=\int \sqrt{\left(1+\frac{\left(-\frac{8 k\left(e^{\alpha t}+\beta\right)^{-2 m}}{A^{2}}-\frac{8 e^{\alpha t} m \alpha^{2}\left(3 e^{\alpha t} m+2 \beta\right)}{\left(e^{\alpha t}+\beta\right)^{2}}+\frac{B d n(\ln t)^{n-2}\left(4 e^{\alpha t} m t \alpha \ln t+B(-1+d) n\left(e^{\alpha t}+\beta\right)(\ln t)^{n}\right)}{t^{2}\left(e^{\alpha t}+\beta\right)}\right)}{\left.t^{2}\left(\frac{e^{2 \alpha t} m^{2} \alpha^{2}}{\left(e^{\alpha t}+\beta\right)^{2}}+\frac{k\left(e^{\alpha t}+\beta\right)^{-2 m}}{A^{2}}\right)-\frac{B d n(\ln t)^{n-2}(4(n-1)-4 \ln t)+B(1+d) n(\ln t)^{n}}{t^{2}}\right)}\right)} d t
$$

Using equation (7), we can find the expressions for $r$ and $s$ as

$$
\begin{gathered}
r=1+\frac{3(d+3)\left(\frac{3 e^{\alpha t} m \alpha^{2} \beta}{\left(e^{\alpha t}+\beta\right)^{2}}+\frac{B d n(\ln t+n-1)(\ln t)^{n-2}}{t^{2}}\right)}{\left(\frac{3 e^{\alpha t} m \alpha}{e^{\alpha t}+\beta}+\frac{B d n(\ln t)^{n-1}}{t}\right)^{2}} \\
+\frac{(d+3)^{2}\left(\frac{3 e^{\alpha t} m \alpha^{3} \beta\left(-e^{\alpha t}+\beta\right)}{\left(e^{\alpha t}+\beta\right)^{3}}+\frac{B d n(\ln t)^{n-3}(2+(-3+n) n+\ln t(3-3 n+2 \ln t))}{t^{3}}\right)}{\left(\frac{3 e^{\alpha t} m \alpha}{e^{\alpha t}+\beta}+\frac{B d n(\ln t)^{n-1}}{t}\right)^{3}} \\
s=-\frac{(d+3)\left(\frac{3 e^{\alpha t} m \alpha^{2} \beta}{\left(e^{\alpha t}+\beta\right)^{2}}+\frac{B d n(\ln t+n-1)(\ln t)^{n-2}}{t^{2}}\right)}{\left(\frac{3 e^{\alpha t} m \alpha}{e^{\alpha t}+\beta}+\frac{B d n(\ln t)^{n-1}}{t}\right)^{2}\left(\frac{3}{2}+\frac{(d+3)\left(\frac{3 e^{\alpha t} m \alpha^{2} \beta}{\left(e^{\alpha t}+\beta\right)^{2}}+\frac{B d n(\ln t+n-1)(\ln t)^{n-2}}{t^{2}}\right)}{\left(\frac{3 e^{\alpha t} m \alpha}{e^{\alpha t}+\beta}+\frac{B d n(\ln t)^{n-1}}{t}\right)^{2}}\right)} \\
-\frac{(d+3)^{2}\left(\frac{3 e^{\alpha t} m \alpha^{3} \beta\left(-e^{\alpha t}+\beta\right)}{\left(e^{\alpha t}+\beta\right)^{3}}+\frac{B d n(\ln t)^{n-3}(2+(-3+n) n+\ln t(3-3 n+2 \ln t))}{t^{3}}\right)}{3\left(\frac{3 e^{\alpha t} m \alpha}{e^{\alpha t}+\beta}+\frac{B d n(\ln t)^{n-1}}{t}\right)^{3}\left(\frac{3}{2}+\frac{(d+3)\left(\frac{3 e^{\alpha t} m \alpha^{2} \beta}{\left(e^{\alpha t}+\beta\right)^{2}}+\frac{B d n(\ln t+n-1)(\ln t)^{n-2}}{t^{2}}\right)}{\left(\frac{3 e^{\alpha t} m \alpha}{e^{\alpha t}+\beta}+\frac{B d n(\ln t)^{n-1}}{t}\right)^{2}}\right)}
\end{gathered}
$$

In figures 5 and 6 we have plotted the tachyonic field potential against $V$ against scalar field $\phi$ for this scenario and $r-s$ trajectory respectively.

\section{- Logamediate-Intermediate Scenario:}


In this section we consider $a$ in the logamediate form and $b$ in the intermediate form. We name this scenario as "logamediate-intermediate Scenario". Hence, in this scenario we consider $a(t)$ and $b(t)$ as follows:

$$
a(t)=\exp \left(A(\ln t)^{m}\right) \text { and } b(t)=\exp \left(B t^{n}\right)
$$

Using equations (4)-(6), we can find the expressions for $V(\phi)$ and $\phi$ as

$$
V(t)=\frac{1}{8}
$$

$$
\begin{aligned}
& \times \sqrt{\left(-\frac{e^{-2 A(\ln t)^{m}}}{t^{4}(\ln t)^{2}}\left(\left(24 k t^{2}-B d e^{2 A(\ln t)^{m}} n t^{n}\left(-4+n\left(4+B(1+d) t^{n}\right)\right)\right)(\ln t)^{2}+24 A^{2} e^{2 A(\ln t)^{m}} m^{2}(\ln t)^{2 m}\right)\right)} \\
& \times \sqrt{\left(-8 e^{-2 A(\ln t)^{m}} k t^{2}+B^{2}(-1+d) d n^{2} t^{2 n}+4 A m(\ln t)^{m-2}\left(4-4 m+\left(4+B d n t^{n}\right) \ln t-6 A m(\ln t)^{m}\right)\right)}
\end{aligned}
$$

$$
\begin{aligned}
& \phi(t)=\int \sqrt{1+\frac{e^{2 A(\ln t)^{m}}(\ln t)^{2}\left(-8 e^{-2 A(\ln t)^{m}} k t^{2}+B^{2}(-1+d) d n^{2} t^{2 n}+4 A m(\ln t)^{m-2}\right.}{\left(24 k t^{2}-B d e^{2 A(\ln t)^{m}} n t^{n}\left(-4+n\left(4+B(1+d) t^{n}\right)\right)\right)(\ln t)^{2}+24 A^{2} e^{2 A(\ln t)^{m} m^{2}(\ln t)^{2 m}}}} \\
& \overline{\times\left(4-4 m+\left(4+B d n t^{n}\right) \ln t-6 A m(\ln t)^{m}\right)} d t
\end{aligned}
$$

Using equation (7), we can find the expressions for $r$ and $s$ as

$$
\begin{gathered}
r=1+\frac{3(d+3)\left(B d(n-1) n t^{n}(\ln t)^{2}+3 A m(-\ln t+m-1)(\ln t)^{m}\right)}{\left(B d n t^{n} \ln t+3 A m(\ln t)^{m}\right)^{2}} \\
+\frac{\left((d+3)^{2}\left(B d n(2+(n-3) n) t^{n}(\ln t)^{3}+3 A m(\ln t)^{m}(2+(m-3) m+\ln t(3-3 m+2 \ln t))\right)\right)}{\left(B d n t^{n} \ln t+3 A m(\ln t)^{m}\right)^{3}}
\end{gathered}
$$

$$
\begin{aligned}
s= & -\frac{\left(( d + 3 ) \left(3\left(B d n t^{n} \ln t+3 A m(\ln t)^{m}\right)\left(B d(-1+n) n t^{n}(\ln t)^{2}+3 A m(-\ln t+m-1)(\ln t)^{m}\right)+(d+3)\right.\right.}{3\left(B d n t^{n} \ln t+3 A m(\ln t)^{m}\right)^{3}\left(\frac{3}{2}+\frac{3(d+3)\left(B d(n-1) n t^{n}(\ln t)^{2}+3 A m(-\ln t+m-1)(\ln t)^{m}\right)}{\left(B d n t^{n} \ln t+3 A m(\ln t)^{m}\right)^{2}}\right)} \\
& \frac{\left.\left.\times\left(B d n(2+(-3+n) n) t^{n}(\ln t)^{3}+3 A m(\ln t)^{m}(2+(-3+m) m+\ln t(3-3 m+2 \ln t))\right)\right)\right)}{}
\end{aligned}
$$

In figures 7 and 8 we have plotted the tachyonic field potential against $V$ against scalar field $\phi$ for this "logamediate-intermediate scenario" and $r-s$ trajectory respectively. 


\section{DISCUSSIONS}

In this work, we have considered $N(=4+d)$-dimensional Einstein's field equations in which 4-dimensional space-time is described by a FRW metric and that of the extra $d$-dimensions by an Euclidean metric. We have considered four scenarios, namely, Emergent-Powerlaw, EmergentIntermediate,Emergent-Logamediate and Logamediate-Intermediate scenarios where the universe is filled with Tachyonic Field dark energy. The natures of the potentials as well as dynamics of scalar fields for the Tachyonic Field dark energy models have been analyzed. The statefinder parameters have been considered and their natures have been investigated for four reconstructed scenarios of the universe.

In the case of Emergent-Power law scenario, we have reconstructed particular forms of scale factors $a$ and $b$ where $a$ belongs to Emergent Scenario and $b$ belongs to Powerlaw scenario in such a way that there is no singularity for evolution of the anisotropic Universe. We have found $\phi$ and potential $V$ in terms of cosmic time $t$ for Tachyonic Field. Here we have shown that the Emergent-Powerlaw scenario is possible for open, closed or flat Universe if the Universe contains Tachyonic Field. From figure 1 it has been seen that the potential always increases with Tachyonic Field. The $\{r, s\}$ diagram (fig.2) shows that the evolution of the Emergent-Powerlaw Universe starts from asymptotic Einstein's static era $(r \rightarrow \infty, s \rightarrow-\infty)$ and goes to $\Lambda$ CDM model $(r=1, s=0)$. It is also observed that $r, s$ are independent of the dimension $d$. So, from statefinder parameters, the behavior of different stages of the evolution of the Emergent-Powerlaw Universe have been generated.

In the case of Emergent-Intermediate scenario, we have reconstructed particular forms of scale factors $a$ and $b$ where $a$ belongs to Emergent Scenario and $b$ belongs to Intermediate scenario in such a way that there is no singularity for evolution of the anisotropic Universe. We have found $\phi$ and potential $V$ in terms of cosmic time $t$ for Tachyonic Field. Here we have shown that the Emergent-Intermediate scenario is possible for open, closed or flat Universe if the Universe contains Tachyonic Field. From figure 3 it has been seen that the potential always increases with Tachyonic Field. The $\{r, s\}$ diagram (fig.4) shows that the evolution of the Emergent-Intermediate Universe starts from asymptotic Einstein's static era $(r \rightarrow \infty, s \rightarrow-\infty)$ and goes to $\Lambda \mathrm{CDM}$ model $(r=1, s=0)$. It is also observed that $r, s$ are independent of the dimension $d$. So, from statefinder parameters, the behavior of different stages of the evolution of 
the Emergent-Intermediate Universe have been generated.

In the case of Emergent-Logamediate scenario, we have reconstructed particular forms of scale factors $a$ and $b$ where $a$ belongs to Emergent Scenario and $b$ belongs to Logamediate scenario in such a way that there is no singularity for evolution of the anisotropic Universe. We have found $\phi$ and potential $V$ in terms of cosmic time $t$ for Tachyonic Field. Here we have shown that the Emergent-Intermediate scenario is possible for open, closed or flat Universe if the Universe contains Tachyonic Field. From figure 5 it has been seen that the potential always increases with Tachyonic Field. The $\{r, s\}$ diagram (fig.6) shows that the evolution of the Emergent-Logamediate Universe starts from asymptotic Einstein's static era $(r \rightarrow \infty, s \rightarrow-\infty)$ and goes to $\Lambda \mathrm{CDM}$ model $(r=1, s=0)$. It is also observed that $r, s$ are independent of the dimension $d$. So, from statefinder parameters, the behavior of different stages of the evolution of the Emergent-Logamediate Universe have been generated.

In the case of Logamediate-Intermediate scenario, we have reconstructed a particular forms of scale factors $a$ and $b$ where $a$ belongs to Logamediate Scenario and $b$ belongs to Intermediate scenario in such a way that there is no singularity for evolution of the anisotropic Universe. We have found $\phi$ and potential $V$ in terms of cosmic time $t$ for Tachyonic Field. Here we have shown that the Logamediate-Intermediate scenario is possible for open, closed or flat Universe if the Universe contains Tachyonic Field. From figure 7 it has been seen that the potential always decreases with Tachyonic Field. The $\{r, s\}$ diagram (fig.8) shows that the evolution of the Logamediate-Intermediate Universe starts from asymptotic Einstein's static era $(r \rightarrow \infty, s \rightarrow-\infty)$ and goes to $\Lambda \mathrm{CDM}$ model $(r=1, s=0)$. It is also observed that $r, s$ are independent of the dimension $d$. So, from statefinder parameters, the behavior of different stages of the evolution of the Logamediate-Intermediate Universe have been generated.

\section{ACKNOWLEDGMENTS}

The authors are thankful to the reviewers for providing constructive and thoughtful suggestions to enhance the quality of the paper. The second author is thankful to the Inter-University Centre for Astronomy and Astrophysics (IUCAA), Pune for providing Visiting Associateship to him. 
[1] Y-F. Cai et al, Physics Reports 493 (2010) 1.

[2] E.J. Copeland, M. Sami, S. Tsujikawa, Internat. J. Modern Phys. D 15 (2006) 1753. arXiv:hep th/0603057.

[3] T. Padmanabhan, Curr. Sci. 88 (2005) 1057.

[4] M. Li et al. arXiv:1103.5870v4 [astro-ph.CO].

[5] B. Ratra and P. J. E. Peebles, Phys. Rev. D 37 (1988) 3406.

[6] S. Nojiri, S. D. Odintsov, Gen. Relativ. Grav. 38(2006)1285.

[7] V. Gorini, A. Kamenshchik, U. Moschella, Phys. Rev. D 67(2003)063509.

[8] L. P. Chimento, Phys. Rev. D 69 (2004) 123517.

[9] W. Zhao, Phys. Lett. B 655 (2007) 97.

[10] N. Arkani-Hamed, S. Dimopoulos and G. Dvali, Phys. Lett. B 429 (1998) 263.

[11] K. Poppenhaeger, S. Hossenfelder, S. Hofmann, M. Bleicher, Phys. Lett. B 582 (2004) 1.

[12] T. Kaluza Preus. Acad. Wiss F1 9669(1921).

[13] O. Klein, A. Phys 37895 (1926).

[14] L. Randall and R. Sundrum, Phys. Rev. Lett 833370 (1999).

[15] P. S. Wesson, World Scientific Space-Time-Matter, (1999).

[16] M. R. Setare, Phys. Lett. B 653, (2007) 116.

[17] M. R. Setare, J. Sadeghi, A. R. Amani, Phys. Lett. B 673 (2009) 241.

[18] N. Arkani-Hamed, S. Dimopoulos, N. Kaloper and J.March-Russel, Nucl. Phys. B 567189 (2000).

[19] D. H. Lyth, Phys. Lett. B 448191 (2002); J. M. Cline, Phys. Rev. D 61023513 (2000).

[20] A. Lukas, B. A. Ovrut and D. Waldram, Phys. Rev. D 61023506 (2000).

[21] H. A. Chamblin and H. S. Reall, Nucl. Phys. B 562133 (1999).

[22] D. Panigrahi and S. Chatterjee, Gen. Rel. Grav. 40833 (2008).

[23] J. Ibánez and E. Vardaguer, Astrophys. J. 306401 (1986).

[24] K. D. Krori, P. Borgohain and K. Das, Gen. Rev. Grav. 22791 (1990).

[25] R. J. Gleiser and M. C. Diaz, Phys. Rev. D 373761 (1988).

[26] B. C. Paul, Phys. Rev. D 64027302 (2001).

[27] D. S. Gorbunov and S. M. Sibiryakov, arXiv:0804.2248 hep-th].

[28] G. Peng et al, Int. J. Theor. Phys. 411127 (2002).

[29] D. Panigrahi and S. Chatterjee, Int. J. Mod. Phys. A 216491 (2006).

[30] G. W. Gibbons, Phys. Lett. B 537(2002)1.

[31] A. Feinstein, Phys. Rev. D 66(2002)063511.

[32] M. Sami, P. Chingangbam and T. Qureshi, Phys. Rev. D 66 (2002) 043530; 
M. Jamil, A. Sheykhi, Int.J. Theor. Phys 50 (2011) 625.

[33] S. Chattopadhyay, U. Debnath, G. Chattopadhyay, Astrophys. Space Sci. 314 (2008) 41.

[34] V. Sahni et al: JETP Lett. 77 (2003) 201.

[35] S. Mukherjee et al. Class. Quantum Grav. 23 (2006) 6927.

[36] J. D. Barrow, N. J. Nunes, Phys. Rev. D 76 (2007) 043501.

[37] G. F. R. Ellis, M. Madsen, Class. Quantum Grav. 8 (1991) 667.

[38] N. Banerjee, S. Das, Gen. Relativ. Grav. 37 (2005) 1695.

[39] M. Sami, Mod. Phys. Lett. A 18(2003) 691.

[40] Y. S. Piao, R. G. Cai, X. M. Zhang and Y. Z. Zhang, Phys. Rev. D 66(2002)121301.

[41] S. Chakraborty and U. Debnath Int J Theor Phys 49(2010) 1693-1698.

[42] S. Chakraborty and U. Debnath Int J Modern Phys A Vol. 25 24(2010) 4691-4701.

[43] S. Chakraborty and U. Debnath Int J Theor Phys 50(2011) 80-87. 